\title{
Massive Pulmonary Emboli Treated with Multidisciplinary Modalities against All Odds: Case Report
}

\author{
Saban $\mathbf{M}^{1,2^{*}}$, Zaretsky $L^{1}$, Salama Rabia ${ }^{1}$, and Darawsha $A^{1,2}$ \\ ${ }^{1}$ Rambam Health Care Campus, Haifa, Israel \\ ${ }^{2}$ The Cheryl Spencer Department of Nursing, University of Haifa, Israel
}

*Corresponding author: Mor Saban, The Cheryl Spencer Department of Nursing, The Faculty of Social Welfare and Health Sciences, University of Haifa, Israel, Tel: +972052030191; E-mail: msaban1@univ.haifa.ac.il

\begin{abstract}
We present a case of an 83 years-old-male who presented to the emergency department at a tertiary hospital with cardiac arrest and was immediately transported to the resuscitation bay. He was diagnosed with respiratory failure, right heart strain and shock. At a later point, after 30 minutes of cardio-pulmonary resuscitation and hemodynamic stabilization with two vasopressors, it was decided to confirm pulmonary embolus diagnosis with $\mathrm{CT}$ angiography. We present a case study with positive results despite faint chances of success due to an aggressive approach to large pulmonary embolus, in part owing to multidisciplinary response team, rapid diagnosis and prompt surgical intervention.
\end{abstract}

Keywords: CT angiography; Pulmonary embolus; Resuscitation; Thrombectomy

Received Date: August 03, 2018; Accepted Date: September 05, 2018; Published Date: September 12, 2018

\section{Introduction}

Diagnosing pulmonary embolus (PE) can be challenging because the signs and symptoms are often non-specific [1]. It carries a grave prognosis and the treatment options are limited [2]. Open surgical thrombectomy is a very high-risk procedure and is many times contraindicated [3]. In general, surgical thrombectomies are reserved for cases of massive PE, as defined by acute PE associated with hemodynamic instability [4]. Despite advances in diagnosis and therapy, acute PE remains associated with a high mortality rate [5].

\section{Case History}

Male, 83-years-old, was transported to the emergency department (ED) by emergency medical service due to loss of consciousness at home. Medical history includes ischemic heart disease, hypertension, diabetes mellitus and peripheral vascular disease. Upon his arrival he was transferred to the resuscitation bay due to loss of consciousness and was pale and sweating. In the resuscitation bay the patient lost pulse. The nurse began cardiopulmonary resuscitation and a resuscitation code was announced. Two minutes later a return of spontaneous circulation (ROSC) was evidenced. Complete right bundle branch was seen on the ECG (Figure 1).

Citation: Mor Saban, Massive Pulmonary Emboli Treated with Multidisciplinary Modalities against All Odds: Case Report. J Clin Cases Rep 2(1): 1-4. DOI: https://doi.org/10.46619/joccr.2019.2-1028

(C) 2019 Tridha Scholars. 


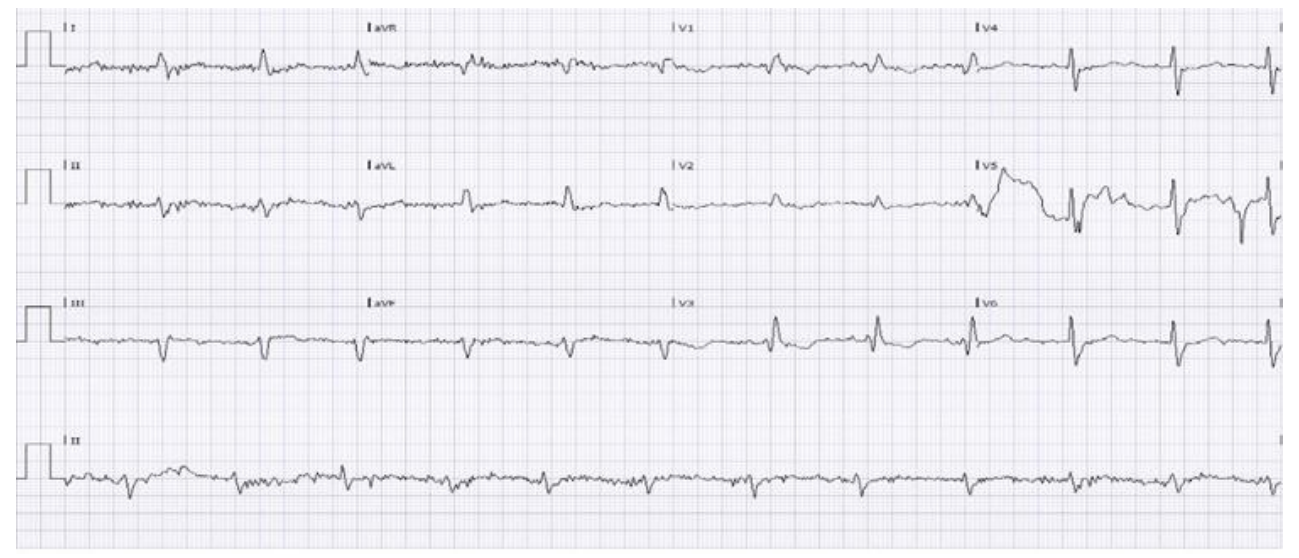

Figure 1: Initial 12-lead electrocardiogram showing signs of right heart strain including right bundle branch block and right axis deviation in patient found to have a large pulmonary embolus.

Seven minutes later resuscitation efforts were renewed due to a loss of pulse. Twenty minutes later ROSC was achieved. After resuscitation attempts the patient's blood pressure remained low and he required a constant titration of Noradrenaline (Levophed) and Dopamine. Bedside echocardiography showed diminished function of right ventricle. Blood test results indicated respiratory and metabolic acidosis (pH: 6.86, PaCO2: 89.0, bicarbonate: 8.6, lactate: 15.0), creatinine: 2.51 and troponin T:0. Despite hemodynamic instability and unclear clinical presentation, it was decided to perform CTA to confirm PE diagnosis. CTA showed bilateral massive PE in both pulmonary arteries (Figure 2).

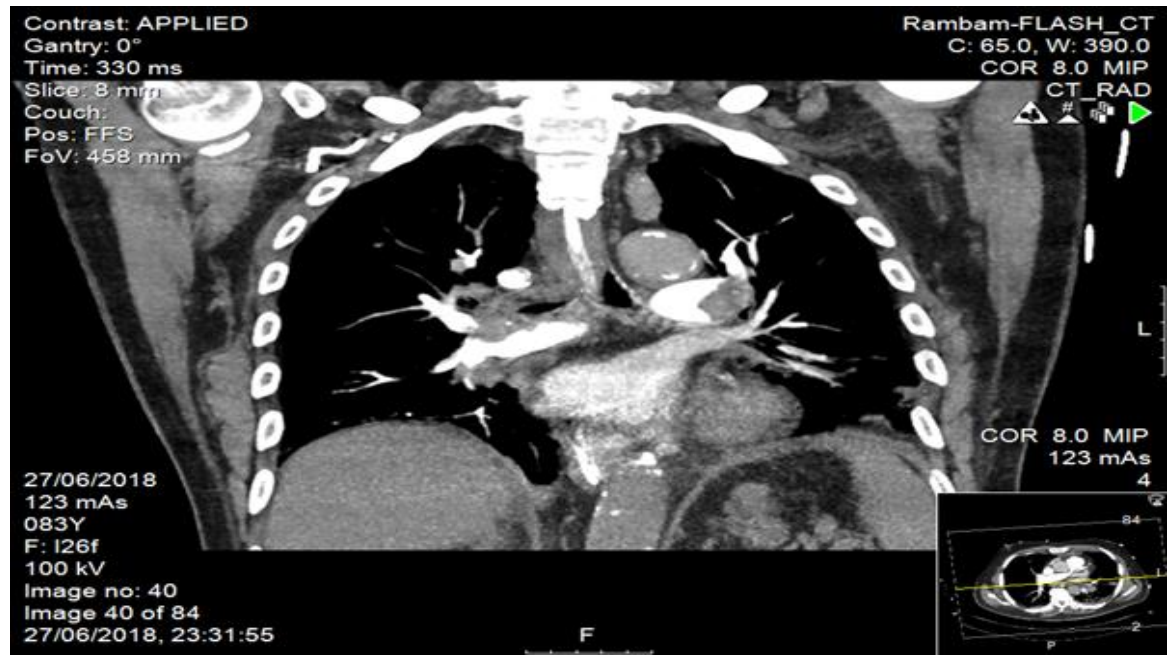

Figure 2: CTA results that demonstrate pulmonary artery occlusion.

TPA or low molecular weight heparin was contraindicated due to resuscitation and cardiogenic shock. Subsequently it was decided to perform emergency thrombectomy (Image III). Afterwards, patient was connected to extracorporeal membrane machine (ECMO) with cardioplegia.

After both emboli were extracted, patient was gradually waned off the ECMO with the help of Adrenalin and a temporary external pacemaker. Later, patient became hemodynamically stable, two chest tubes remained, and he was transferred to chest surgery intensive care unit. That evening the patient was extubated and received continues positive airway pressure. Patient was discharged four days later with no permanent complications. 


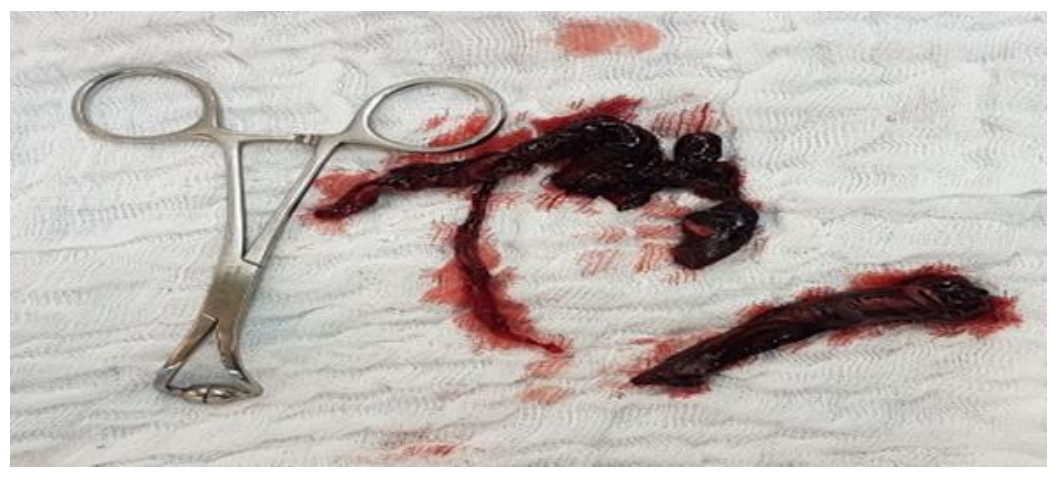

Figure 3: Thrombus after the extraction from the pulmonary artery.

\section{Discussion}

Treatment of massive PE with cardiogenic shock remains challenging with high mortality rates, especially among older adults with risk factors [1]. A sentinel element in the chain of events was the fast response of the resuscitation bay nurse [6]. The biggest task is to identify a PE while patient is in cardiac arrest with unclear symptoms. In this case, the echocardiography was the only asset that contributed directly to the suspicion of PE [7]. The next phase, a definite diagnosis in the form of CTA was considered carefully due to the hemodynamic instability [8]. This dilemma occurs consistently while patient is unstable and there is a clinical uncertainty whether he needs surgery or further gold standard imaging [9].

\section{Conclusion}

Multidisciplinary care in patients with life-threatening conditions is a corner stone in the ED. This case report shows how team resolve to save lives can be expressed in extreme situations such as massive PE and result in complete success.

\section{References}

1. Sharif S, Eventov M, Kearon C, et al. (2018) Comparison of the age-adjusted and clinical probability-adjusted D-dimer to exclude pulmonary embolus in the emergency department. American Journal of Emergency Medicine S07356757(18): 30633-30638.

2. Yusuff H, Zochios V, Vuylsteke A (2015) Extracorporeal membrane oxygenation in acute massive pulmonary embolus: a systematic review. Perfusion 30: 611-616.

3. Pollack CV, Schreiber D, Goldhaber SZ, et al. (2011) Clinical characteristics, management, and outcomes of patients diagnosed with acute pulmonary embolus in the emergency Department: Initial Report of EMPEROR (Multicenter Emergency Medicine Pulmonary Embolism in the Real World Registry). Journal of the American College of Cardiology 57(8): 700-706.

4. Fong J, Santikul D, Liu T (2018) Right heart thrombus with acute pulmonary embolus from a massive deep vein thrombosis. Visual Journal of Emergency Medicine 10: 122-124.

5. Leacche M, Unic D, Goldhaber SZ, et al. (2005) Modern surgical treatment of massive pulmonary embolus: Results in 47 consecutive patients after rapid diagnosis and aggressive surgical approach. The Journal of Thoracic and Cardiovascular Surgery 129(5): 1018-1023.

6. Dami F, Heymann E, Pasquier M, et al. (2015) Time to identify cardiac arrest and provide dispatch-assisted cardiopulmonary resuscitation in a criteria-based dispatch system. Resuscitation 97: 27-33.

7. Vanni S, Nazerian P, Bova C, et al. (2017) Comparison of clinical scores for identification of patients with pulmonary embolus at intermediate-high risk of adverse clinical outcome: the prognostic role of plasma lactate. Internal and 
http://www.tridhascholars.org | January-2019

Emergency Medicine 12(5): 657-665.

8. Huber-Wagner S, Biberthaler P, Häberle S, et al. (2013) Whole-Body CT in haemodynamically unstable severely injured patients - A retrospective, multicentre study. PLoS One 8: e68880.

9. Jiang L, Ma Y, Jiang S, et al. (2014) Comparison of whole-body computed tomography vs selective radiological imaging on outcomes in major trauma patients: a meta-analysis. Scandinavian Journal of Trauma, Resuscitation and Emergency Medicine 22: 54. 